\title{
The emergence of magnetic flux through a partially ionised solar atmosphere
}

\author{
J. E. Leake and T. D. Arber
}

Space and Astrophysics group, Dept. of Physics, University of Warwick, Gibbet Hill Road, CV4 7AL, Coventry, UK

e-mail: J.E.Leake@warwick.ac.uk

Received 24 August 2005 / Accepted 16 January 2006

\section{ABSTRACT}

We present results from 2.5D numerical simulations of the emergence of magnetic flux from the upper convection zone through the photosphere and chromosphere into the corona. Certain regions of the solar atmosphere are at sufficiently low temperatures to be only partially ionised, in particular the lower chromosphere. This leads to Cowling resistivities orders of magnitude larger than the Coulomb values, and thus to anisotropic dissipation in Ohm's law. This also leads to localised low magnetic Reynolds numbers $\left(R_{\mathrm{m}}<1\right)$. We find that the rates of emergence of magnetic field are greatly increased by the partially ionised regions of the model atmosphere, and the resultant magnetic field is more diffuse. More importantly, the only currents associated with the magnetic field to emerge into the corona are aligned with the field, and thus the newly formed coronal field is force-free.

Key words. magnetohydrodynamics (MHD) - magnetic fields - plasmas - Sun: atmosphere - Sun: sunspots - Sun: magnetic fields

\section{Introduction}

The evolution of the solar atmosphere, in particular the corona, is strongly influenced by the presence of magnetic field. This coronal field is believed to originate beneath the surface, where dynamo action at the base of the convection zone can generate large scale toroidal fields (Spiegel \& Weiss 1980; Hughes \& Proctor 1988; Gilman et al. 1989). The instabilities present in these fields gives rise to the formation of magnetic flux tubes which are driven by buoyancy through the convection zone up to the surface and emerge into the atmosphere above. This process is known as flux emergence. Flux emergence is widely regarded as the process by which new active regions on the Sun's surface are formed. The emerging fields evolve to form the complex structures we see in the Sun's atmosphere, such as coronal loops and prominences, and strongly influence dynamic events such as flares and coronal mass ejections.

Emerging magnetic field in the photosphere has been measured by MDI. Vector magneto-grams have been used to reconstruct the resultant 3D magnetic field in the corona (Berton 2000). Associated with the observed emergence at photospheric levels bright coronal loops are observed in the corona, as detected by the SOHO and TRACE X-ray and EUV instruments. These loops appear to have foot-points of opposite polarity in the active region below.

Following the work of Parker $(1954,1979)$ which showed that the instability of large scale magnetic field at the base of the convection zone could lead to sunspot formation, there has been a wealth of theoretical and numerical studies concerning the evolution of magnetic flux tubes in both the convection zone and the atmosphere above.

These magnetic fields held in the overshoot region of the solar interior can be generated to strengths of up to kG by dynamo actions and once the field strengths exceed $10^{5} \mathrm{G}$ undular instabilities lead to the formation of loops which rise up to the surface on time-scales of months (Moreno-Insertis 1986; D'Silva \& Choudhuri 1993; Caligari et al. 1995; Fan et al. 1994).

Emonet \& Moreno-Insertis (1998) investigated the effect of surrounding flows on the flux tube's rise to the surface and demonstrated that a minimum twist was required in the tubes in order to suppress the conversion of the tube into vortex pairs. Fan et al. (1998) investigated flux tubes unstable to the $m=1$ kink instability in the convection zone. For flux tubes to survive the crossing of the convection zone their twist must be large enough to avoid fragmentation but small enough to not be kink unstable. This gives a range of twist for flux tubes in order that they survive the rise though the convection zone. However, the kink instability may also present a mechanism for emergence through the photosphere, once the magnetic field has reached the top of the convection zone.

The emergence of magnetic field from the photosphere into the atmosphere above has also been studied extensively in recent years (Matsumoto \& Shibata 1992; Matsumoto et al. 1993; Magara \& Longcope 2001; Shibata et al. 1989). These authors studied the emergence of magnetic field held at the photosphere by the convectively stable stratification. It was shown that these 
fields were unstable to mixed modes of the magnetic buoyancy instability. The resulting expansion of magnetic field was shown to match certain observational data of active regions. Nozawa et al. (1992), and Matsumoto et al. (1993) modified the analysis by looking at the effect of convection on the instability, showing how field held beneath the photosphere could emerge into the atmosphere.

More recent simulations have combined the buoyant and expansive phases of the emergence process, simulating rising magnetic flux tubes in the convection zone followed by the expansion through the photosphere into the corona via the magnetic buoyancy instability (Fan 2001; Magara \& Longcope 2003; Manchester 2001). In these simulations an isolated twisted flux tube is inserted into the upper part of the convection zone which rises by magnetic buoyancy until it reaches the stable layers of the photosphere. The resulting, predominantly horizontal, expansion leads to the formation of a magnetic layer which is unstable to the magnetic buoyancy instability and this drives magnetic field into the atmosphere above.

Archontis et al. (2004) and Galsgaard et al. (2005) showed how this emerging field would interact with a pre-existing coronal field, the formation of current sheets and jets occurring as a by-product of reconnection. This reconnection with coronal field helps the flux tube to emerge through the lower atmosphere as field lines from beneath the surface are reconnected to coronal field lines.

All these numerical simulations use a standard model for the solar atmosphere. The model consists of an adiabatically stratified convection zone, isothermal photosphere/chromosphere, isothermal corona and a transition region between the two isothermal regions. Furthermore the simulations are performed under the MHD approximation. Under this assumption the atmosphere of the Sun is represented by a fully ionised plasma, consisting of electrons and ions only. Under this and other limiting assumptions the governing set of equations are:

$$
\begin{aligned}
\frac{\partial \rho}{\partial t} & =-\nabla \cdot(\rho \boldsymbol{v}) \\
\frac{\partial}{\partial t}(\rho \boldsymbol{v}) & =-\nabla \cdot(\rho \boldsymbol{v} \boldsymbol{v})+(\boldsymbol{j} \wedge \boldsymbol{B})-\nabla P+\rho \boldsymbol{g}+\nabla \cdot \boldsymbol{S} \\
\frac{\partial \boldsymbol{B}}{\partial t} & =\nabla \wedge(\boldsymbol{v} \wedge \boldsymbol{B})-\nabla \wedge(\eta \boldsymbol{j}) \\
\frac{\partial}{\partial t}(\rho \boldsymbol{\epsilon}) & =-\nabla \cdot(\rho \boldsymbol{\epsilon})-P \nabla \cdot \boldsymbol{v}+\eta j^{2}+\varsigma_{i j} S_{i j}
\end{aligned}
$$

where $\rho$ is the mass density, $P$ is the gas pressure, $\epsilon$ is the internal specific energy density, $\boldsymbol{v}$ is the bulk velocity of the plasma, $\boldsymbol{B}$ the magnetic field, and $\boldsymbol{j}$ the current density. $\boldsymbol{g}$ is gravitational acceleration, and $\eta$ is the resistivity. $\boldsymbol{S}$ is the stress tenor which has components $S_{i j}=v\left(\varsigma_{i j}-\frac{1}{3} \delta_{i j} \nabla \cdot v\right)$, and $\varsigma_{i j}=\frac{1}{2}\left(\frac{\partial v_{\mathrm{i}}}{\partial x_{j}}+\frac{\partial v_{j}}{\partial x_{\mathrm{i}}}\right)$. Here we are operating in the S.I. unit system. These four equation are closed with an equation of state.

This paper aims to improve this basic model of flux emergence in two distinct ways. Firstly the governing equations take into account the partial ionisation of certain regions of the solar atmosphere. The lower chromosphere of the Sun's atmosphere is partially ionised, and depending on the local magnetic field can be weakly ionised (Khodachenko et al. 2004). This is not taken into account in the standard MHD model. The effect of the possible presence of neutrals in a plasma on the governing equations is investigated, and a new set of equations is derived to simulate flux emergence through the lower atmosphere of the Sun. As the presence of neutrals will affect momentum transfer due to relative velocities, we expect the emergence and evolution of magnetic field to be affected by these improvements to the model. We present results on the rates of flux emergence and the nature of emerging flux tubes as they pass through the partially ionised region of the atmosphere, as well as the resultant structure of the fields.

Secondly, we model the heat transfer mechanisms present in the solar atmosphere that cannot be simulated directly. The above equation for energy includes only adiabatic terms (representing no heat transfer), a Joule heating term from ion-electron collisions, and viscous terms. Thus in the current models the reaction of the atmospheric plasma to the emergence of magnetic fields is unrealistic in that none of the heating terms, such as radiative losses, thermal conduction and shock dissipation of short scale, unresolved acoustic modes are present (Wedemeyer 2004). We present a simple way of modeling these mechanisms which results in a more realistic reaction of the atmosphere to flux emergence.

The results shown here are for 2.5D simulations. A complete treatment must be 3D in order to capture the full dynamics associated with the emergence of magnetic fields into the solar atmosphere. However, the implementation of the methods included in this work are simpler in $2.5 \mathrm{D}$ and act as a prelude to future 3D work. The restricted geometry adopted here is still capable of demonstrating the importance of partially ionised layers and improved energy equation modeling.

The structure of the paper is as follows. Section 2 describes the theoretical basis for this work and the derivation of the governing equations, for both the partial ionisation of the atmosphere (2.1) and the non-adiabatic terms in the energy Eq. (2.2). Section 3 describes the numerical code (3.1), implementing the Eqs. (3.2) and (3.3), and the model used (3.4). Section 4 presents the results, firstly when the non-adiabatic heating terms are modelled (4.1), and then for the inclusion of partial ionisation in the model atmosphere (4.2). Section 5 contains summaries and conclusions of the work.

\section{Section 2}

\subsection{Partially ionised plasma in the lower solar atmosphere}

The MHD equations given in the introduction are the equations for a fully ionised plasma consisting of ions and electrons. The simplified Ohm's law is used to derive the induction equation for the magnetic field. In this paper all equations used are given in SI units unless stated otherwise.

$$
\boldsymbol{E}+\boldsymbol{v} \wedge \boldsymbol{B}=\frac{1}{\sigma} \frac{\nabla \wedge \boldsymbol{B}}{\mu_{0}}=\frac{1}{\sigma} \boldsymbol{j}
$$

where $\boldsymbol{j}=\nabla \wedge \boldsymbol{B} / \mu_{0}$ is the current density and $\sigma$ is the conductivity. This is the one-fluid approach to a fully ionised plasma 
of ions and electrons. The Hall term and electron pressure term have all been neglected. Combining this with Faraday's law

$\frac{\partial \boldsymbol{B}}{\partial t}=-\nabla \wedge \boldsymbol{E}$

gives the equation for the evolution of the magnetic field.

$\frac{\partial \boldsymbol{B}}{\partial t}=\nabla \wedge(\boldsymbol{v} \wedge \boldsymbol{B})-\nabla \wedge(\eta \boldsymbol{j})$

where $\eta=1 / \sigma$ is known as the resistivity. Diffusion of magnetic field is assumed to be due to collisions of electrons and ions. However the lower atmosphere of the Sun, where the temperature is below the ionisation temperature of hydrogen, is not fully ionised and in some places is weakly ionised. This means that neutrals must be included in the equations describing the plasma.

In order to simulate the solar atmosphere the MHD equations must be modified to include the possibility of the presence of neutral hydrogen. This involves recasting Ohm's law to include the momentum transfer due to collisions with neutrals.

The generalised Ohm's law for a fluid containing ions, electrons and neutral atoms is given by (Cowling 1957; Braginskii 1965)

$$
\begin{aligned}
\boldsymbol{E}+(\boldsymbol{v} \wedge \boldsymbol{B})= & \frac{1}{\sigma} \frac{\nabla \wedge \boldsymbol{B}}{\mu_{0}} \\
& -\frac{\xi_{\mathrm{n}}^{2}}{\alpha_{\mathrm{n}}}\left[\frac{((\nabla \wedge \boldsymbol{B}) \wedge \boldsymbol{B}) \wedge \boldsymbol{B}}{\mu_{0}}\right] \\
= & \frac{1}{\sigma} \boldsymbol{j}+\frac{\xi_{\mathrm{n}}^{2} B_{0}^{2}}{\alpha_{\mathrm{n}}} \boldsymbol{j}_{\perp}
\end{aligned}
$$

where $\boldsymbol{v}$ is the fluid velocity, $\boldsymbol{B}$ is the magnetic field, and $B_{0}$ its magnitude. $\boldsymbol{E}$ is the electric field, and

$\boldsymbol{j}_{\perp}=\frac{\boldsymbol{B} \wedge(\boldsymbol{j} \wedge \boldsymbol{B})}{|\boldsymbol{B}|^{2}}$

is the component of the current density perpendicular to the magnetic field.

The conductivity is defined by

$\sigma=\frac{n_{\mathrm{e}} e^{2}}{m_{\mathrm{e}}\left(v_{\mathrm{ei}}^{\prime}+v_{\mathrm{en}}^{\prime}\right)}$

and

$\alpha_{\mathrm{n}}=m_{\mathrm{e}} n_{\mathrm{e}} v_{\text {en }}^{\prime}+m_{\mathrm{i}} n_{\mathrm{i}} v_{\text {in }}^{\prime}$.

The number densities of species (ion, electron, neutral) are given by $n_{\mathrm{i}}, n_{\mathrm{e}}, n_{\mathrm{n}}$ respectively, and the masses by $m_{\mathrm{i}}, m_{\mathrm{e}}, m_{\mathrm{n}}$.

$\xi_{\mathrm{n}}=\frac{m_{\mathrm{n}} n_{\mathrm{n}}}{m_{\mathrm{n}} n_{\mathrm{n}}+m_{\mathrm{i}} n_{\mathrm{i}}}$

is the relative density of neutrals, and $v_{\text {ie }}^{\prime}, v_{\text {in }}^{\prime}$ and $v_{\text {en }}^{\prime}$ are the effective collisional frequencies defined by

$v_{k l}^{\prime}=\frac{m_{l}}{m_{l}+m_{k}} v_{k l}$ where $k=\mathrm{e}, \mathrm{i}, l=\mathrm{i}, \mathrm{n}$. Following the example of Spitzer (1962), the collisional frequencies of ions and electrons with neutrals are estimated by

$v_{\mathrm{in}}=n_{\mathrm{n}} \sqrt{\frac{8 K_{\mathrm{B}} T}{\pi m_{\mathrm{in}}}} \Sigma_{\mathrm{in}}$
$v_{\mathrm{en}}=n_{\mathrm{n}} \sqrt{\frac{8 K_{\mathrm{B}} T}{\pi m_{\mathrm{en}}}} \Sigma_{\mathrm{en}}$

where

$m_{\mathrm{in}}=\frac{m_{\mathrm{i}} m_{\mathrm{n}}}{m_{\mathrm{i}}+m_{\mathrm{n}}}$

and $K_{\mathrm{B}}$ is Boltzmann's constant. The collisional frequency of electrons and ions is given by

$v_{\mathrm{ei}}=3.7 \times 10^{-6} \frac{n_{\mathrm{i}} \Lambda Z^{2}}{T^{3 / 2}}$

(Spitzer 1962). $\Sigma_{\text {in }}=5 \times 10^{-19} \mathrm{~m}^{2}$ and $\Sigma_{\text {en }}=10^{-19} \mathrm{~m}^{2}$ are the ion-neutral and electron-neutral cross-sections respectively. Here $\Lambda$ is the Coulomb logarithm.

In the generalised Ohm's law (9) the pressure term has been neglected, as the chromospheric plasma is relatively cold, and the Hall term is also neglected. The Hall term can be dropped from the generalised Ohm's law if the plasma is magnetised, i.e. if the ions and electrons are tightly bound to the magnetic field. Khodachenko \& Zaitsev (2002) showed that in the upper photosphere where the plasma is more weakly ionised the positive ions may not be magnetised, i.e. not tightly bound to the magnetic field for certain field strengths. In these simulations the Hall term is neglected throughout. The justification for this approach is shown in Sect. 4, where for a typical simulation the Hall term is compared to the other terms in the generalised Ohm's law.

If the plasma is assumed to be entirely composed of hydrogen then the expressions can be simplified by taking $m_{\mathrm{in}}=$ $m_{\mathrm{n}} / 2, \xi_{\mathrm{n}}=\rho_{\mathrm{n}} / \rho$ resulting in

$\alpha_{\mathrm{n}}=\frac{1}{2} \xi_{\mathrm{n}}\left(1-\xi_{\mathrm{n}}\right) \frac{\rho^{2}}{m_{\mathrm{n}}} \sqrt{\frac{16 k_{\mathrm{B}} T}{\pi m_{\mathrm{i}}}} \Sigma_{\text {in }}$.

Using the definition for the Cowling conductivity as

$\sigma_{\mathrm{c}}=\frac{\sigma}{1+\frac{\xi_{\mathrm{n}}{ }^{2} B_{0}^{2} \sigma}{\alpha_{\mathrm{n}}}}$

and defining the Coulomb and Cowling resistivity as $\eta=1 / \sigma$ and $\eta_{\mathrm{c}}=1 / \sigma_{\mathrm{c}}$ respectively, it is trivial to show that

$\frac{\xi_{\mathrm{n}}^{2} B_{0}^{2}}{\alpha_{\mathrm{n}}}=\eta_{\mathrm{c}}-\eta$.

Thus Eq. (9) can be be written as

$$
\begin{aligned}
\boldsymbol{E}+(\boldsymbol{v} \wedge \boldsymbol{B}) & =\eta \boldsymbol{j}+\left(\eta_{\mathrm{c}}-\eta\right) \boldsymbol{j}_{\perp} \\
& =\eta \boldsymbol{j}_{\|}+\eta_{\mathrm{c}} \boldsymbol{j}_{\perp}
\end{aligned}
$$

and the frictional Joule heating term for this plasma (Braginskii $1965)$ is then given by

$$
\begin{aligned}
Q & =(\boldsymbol{E}+(\boldsymbol{v} \wedge \boldsymbol{B})) \cdot \boldsymbol{j} \\
& =\eta j_{\|}{ }^{2}+\eta_{\mathrm{c}} j_{\perp}{ }^{2}
\end{aligned}
$$


with $\boldsymbol{j}_{\perp}$ and $\boldsymbol{j}_{\|}$being the components of $\boldsymbol{j}$ perpendicular and parallel to $\boldsymbol{B}$.

The induction Eq. (7) now becomes

$$
\begin{aligned}
\frac{\partial \boldsymbol{B}}{\partial t}= & \nabla \wedge(\boldsymbol{v} \wedge \boldsymbol{B})-\nabla \wedge(\eta \boldsymbol{j}) \\
& -\nabla \wedge\left(\eta_{\mathrm{c}}-\eta\right)\left(\boldsymbol{j}_{\perp}\right) \\
\frac{\partial \boldsymbol{B}}{\partial t}= & \nabla \wedge(\boldsymbol{v} \wedge \boldsymbol{B})-\nabla \wedge\left(\eta \boldsymbol{j}_{\|}\right) \\
& -\nabla \wedge\left(\eta_{\mathrm{c}} \boldsymbol{j}_{\perp}\right) .
\end{aligned}
$$

The generalised Ohms law used here (Eq. (22)) neglects the electron pressure and Hall terms. In this approximation, where ions and electrons are tightly bound to the magnetic field, the only difference to Ohm's law for a fully ionised plasma is in the resistivities parallel and perpendicular to the field. The $(\boldsymbol{j} \wedge \boldsymbol{B}) \wedge \boldsymbol{B}$ term corresponds to a genuine increase in the resistivity normal to the field, and if the neutral fraction is appreciable dominates over the other terms (Cowling 1957). This manifests in the above equation for the evolution of the magnetic field. Note that due to the anisotropic nature of the dissipation mechanism, i.e. $\eta_{\mathrm{c}} \gg \eta$, it is not possible to cast the equation for the evolution of magnetic field, or that for the current density, in the form of a simple diffusion equation.

Inserting the joule heating into the energy Eq. (4) gives

$$
\begin{aligned}
\frac{\partial}{\partial t}(\rho \epsilon)= & -\nabla \cdot(\rho \epsilon \boldsymbol{v})-P \nabla \cdot \boldsymbol{v} \\
& +\eta j_{\|}{ }^{2}+\eta_{\mathrm{c}}{j_{\perp}}^{2}+\varsigma_{i j} S_{i j} .
\end{aligned}
$$

The equations for time variation of the bulk plasma velocity and the continuity equation (Eq. (1)) remain unchanged. So the system of equations for a hydrogen plasma with any degree of ionisation is

$$
\begin{aligned}
\frac{\partial \rho}{\partial t}= & -\nabla \cdot(\rho \boldsymbol{v}) \\
\frac{\partial}{\partial t}(\rho \boldsymbol{v})= & -\nabla \cdot(\rho \boldsymbol{v} \boldsymbol{v})+(\boldsymbol{j} \wedge \boldsymbol{B})-\nabla P+\rho \boldsymbol{g}+\nabla \cdot \boldsymbol{S} \\
\frac{\partial \boldsymbol{B}}{\partial t}= & \nabla \wedge(\boldsymbol{v} \wedge \boldsymbol{B})-\nabla \wedge\left(\eta \boldsymbol{j}_{\|}\right) \\
& -\nabla \wedge\left(\eta_{\mathrm{c}} \boldsymbol{j}_{\perp}\right) \\
\frac{\partial}{\partial t}(\rho \boldsymbol{\epsilon})= & -\nabla \cdot(\rho \boldsymbol{\epsilon} \boldsymbol{v})-P \nabla \cdot \boldsymbol{v} \\
& +\eta j_{\|}^{2}+\eta_{\mathrm{c}} j_{\perp}^{2}+\varsigma_{i j} S_{i j} .
\end{aligned}
$$

When the plasma is fully ionised there are no neutrals so $\xi_{\mathrm{n}}=0$, $\eta_{\mathrm{c}}=\eta$ and the equations return to the standard non-ideal MHD equations. Where the plasma is not fully ionised we must evaluate $\eta_{\mathrm{c}}$. In order to evaluate the expression for the Cowling conductivity $\eta_{\mathrm{c}}$ an estimate for the neutral fraction $\xi_{\mathrm{n}}$ is required as a function of density and temperature. Following the method of De Pontieu (1999) an electro-neutral hydrogen plasma is assumed.

The solar chromosphere is not in LTE, hence a simple onelevel model for the hydrogen atom is inadequate for these conditions (Pottasch \& Thomas 1959). A two-level model is used instead for the hydrogen atom, as this is a good approximation to the hydrogen ionisation at chromospheric densities and temperatures (Thomas \& Athay 1961). Under this approximation the ionisation equation (Brown 1973) is

$n \frac{\partial x}{\partial t}=\sum\left(C_{j}+P_{j}+C_{j}^{*}+P_{j}^{*}\right)$

where $n$ is the local number density and $x$ is the ionisation degree. $C_{j}$ and $P_{j}$ are the ionisation rates from level $j$ due to thermal collisions and local radiation field respectively. $C_{j}{ }^{*}$ and $P_{j}{ }^{*}$ are the ionisation rates by non-thermal particles and external radiation field. In the first approximation photoionisation from level 2 to level 1 is provided by the external field alone, so that $P_{1}=P_{1}{ }^{*}=0$. This external field is simply the photospheric radiation field at temperature $T_{\mathrm{R}}$, diluted by a factor $w$ (Brown 1973). The ionisation equation can be further simplified by noting that thermal collision ionisation is unimportant when compared to photoionisation (Ambartsuyan 1958). Thus the ionisation equation is balanced by photoionisation from level 2 to level 1 and spontaneous recombination for the return route.

$n \frac{\partial x}{\partial t}=P_{2_{+}}{ }^{*}-P_{2-}$.

The steady state solution to this equation is given by (Thomas \& Athay 1961)

$$
\begin{aligned}
\frac{n_{\mathrm{i}}{ }^{2}}{n_{\mathrm{n}}} & =\frac{f(T)}{b(T)} \\
f(T) & =\frac{\left(2 \pi m_{\mathrm{e}} K_{\mathrm{B}} T\right)^{\frac{3}{2}}}{h^{3}} \exp \left(-\frac{X_{\mathrm{i}}}{K_{\mathrm{B}} T}\right) \\
b(T) & =\frac{T}{w T_{\mathrm{R}}} \exp \left[\frac{X_{\mathrm{i}}}{4 K_{\mathrm{B}} T}\left(\frac{T}{T_{\mathrm{R}}}-1\right)\right]
\end{aligned}
$$

where $T_{\mathrm{R}}$ is the temperature of the photospheric radiation field and $w=0.5$ is its dilution factor. Using this equation, the ratio of the number density of neutrals to ions is given by

$r=\frac{n_{\mathrm{n}}}{n_{\mathrm{i}}}=\frac{1}{2}\left(-1+\sqrt{\left(1+\frac{4 \rho / m_{\mathrm{i}}}{n_{\mathrm{i}}^{2} / n_{\mathrm{n}}}\right)}\right)$

and the neutral fraction $\xi_{\mathrm{n}}=\rho_{\mathrm{n}} / \rho$ is

$\xi_{\mathrm{n}}=\frac{r}{1+r}$

where $m_{\mathrm{i}}=m_{\mathrm{n}}$ has been used as the atmosphere is assumed to be pure hydrogen. So from the plasma variables $\rho, T$ and $\boldsymbol{B}$ the neutral fraction and thus the value of $\eta_{\mathrm{c}}$ can be calculated.

To demonstrate how the profile of $\eta_{\mathrm{c}}$ looks for the quiet Sun, the solar atmospheric plasma variables given by the VAL C model (Vernazza et al. 1981) are used to calculate the neutral fraction $\xi_{\mathrm{n}}$ and $\eta_{\mathrm{c}}$ for a given magnetic field profile as a function of height. For this calculation the magnetic field model used is that of a vertical expanding flux tube representing magnetic field in the chromosphere (Martinez-Pillet et al. 1997). The form is given by

$|\boldsymbol{B}|=B_{\mathrm{ph}}\left(\frac{\rho}{\rho_{\mathrm{ph}}}\right)^{\alpha}$

with an exponent value of $\alpha=0.3$. The strength falls from $1000 \mathrm{G}$ at $1000 \mathrm{~km}$ to $10 \mathrm{G}$ at $3000 \mathrm{~km}$ above the surface. The profile of $\eta_{\mathrm{c}}$ is shown in Fig. 1, it has a maximum of 


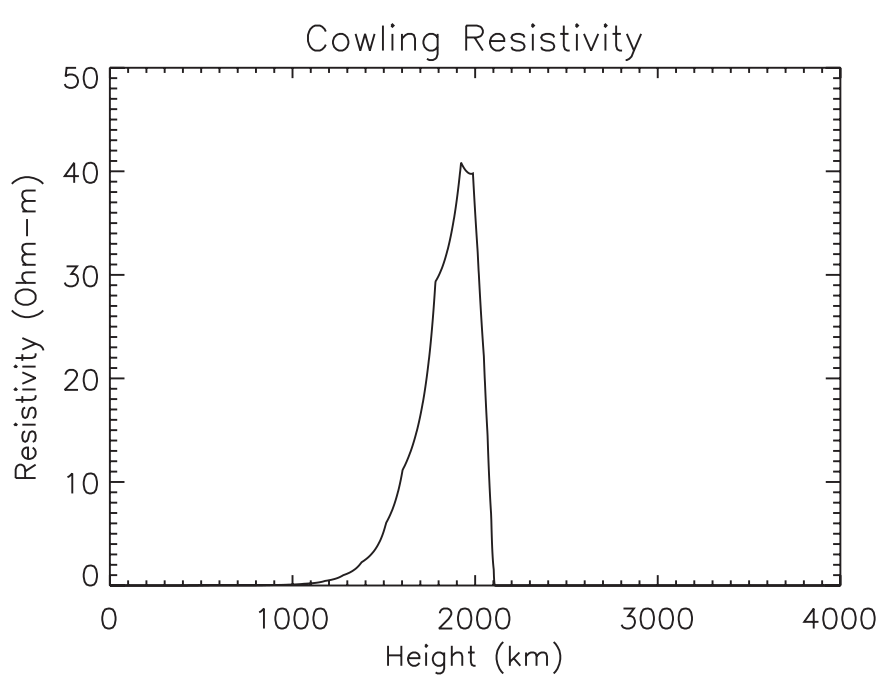

Fig. 1. The Cowling conductivity as a function of height using the VALC model for density and temperature and a magnetic field strength using Eq. (38) and an exponent of $\alpha=0.3$.

approximately $40 \Omega \mathrm{m}$ at a height of $2000 \mathrm{~km}$. A typical value of $\eta$ at this height is of the order of $10^{-10} \Omega \mathrm{m}$ (Khodachenko et al. 2004).

\subsection{The energy equation}

The energy equation used thus far (Eq. (4)) has only included the adiabatic terms and the joule heating term. To simulate the solar atmosphere realistically the many other heat transfer effects that are present must be included.

$\frac{\mathrm{D} \epsilon}{\mathrm{D} t}=-\frac{P}{\rho} \nabla \cdot \boldsymbol{v}+\eta j_{\|}^{2}+\eta_{\mathrm{c}} j_{\perp}^{2}+\varsigma_{i j} S_{i j}+S_{\mathrm{h}}-\nabla . \boldsymbol{q}-L_{\mathrm{R}}+H$.

The non-adiabatic terms on the right hand side include the Joule heating, as derived in Sect. 2.1. The other non-adiabatic terms are viscous heating, shock heating, thermal conduction, radiative transfer and the unknown coronal heating term. Thermal conduction is given by $\nabla . q$, where $\boldsymbol{q}=\kappa \nabla T$ is the heat flux, and $\kappa$ the thermal conductivity. $S_{\mathrm{h}}$ is small scale shock dissipation of acoustic waves present in the chromosphere. These waves are generated by convective motions at the top of the convection zone and propagate upwards, and due to the stratification, shock, dissipate and heat the local plasma (Narain \& Ulmschneider 1990). $L_{\mathrm{R}}$ represents all radiative losses. As well as these diagnosed heating effects there are many others associated with the coronal heating problem, which include reconnection, and the propagation and dissipation of MHD waves, represented here by $H$.

All these heating effects give the observed temperatures in the solar atmosphere, and thus a complete numerical study must include them to accurately simulate the emergence of magnetic flux from the convection zone through the photosphere, chromosphere and into the corona. However, simulating these effects directly is extremely problematic. Small scale effects are difficult to include in large scale MHD simulations. Although effects such as thermal conductivity could be added to the governing equations, the undiagnosed effects such as those associated with coronal heating, which are not known explicitly would be almost impossible to simulate directly.

For this reason a simplified approach to modeling these effects is suggested, following the work of Abbet \& Fisher (2003). The non-adiabatic heating terms mentioned above act to force the temperature profile of the Sun to that which is observed. Rather than include the terms individually it would be simpler to model their effects, namely that of forcing the temperature to the observed profile, by applying a forcing or relaxation term to the temperature profile. Thus when the local temperature of the plasma deviates form the pre-defined value, it is forced back to this pre-defined value, on a time-scale which reflects the different mechanisms of heating/cooling present locally. This can be done by a simple Newton-cooling equation, which given in terms of the specific energy density, $\epsilon$, is

$\frac{\mathrm{d} \epsilon}{\mathrm{d} t}=-\frac{\epsilon-\epsilon_{0}(\rho)}{\tau}$

where $\tau$ is the time-scale of the relaxation. The equilibrium specific energy density $\epsilon_{0}$ is chosen to be a function of the density $\rho$. The reasoning for this is related to the nature of these simulations. The buoyancy force drives magnetic field in the convection zone upwards into the photosphere, where the field then expands into the atmosphere above. Thus as a parcel of plasma from the convection zone of density $\rho$ is moved upwards into the photosphere, its temperature should be relaxed to its own initial temperature, rather than the local plasma temperature, which is of a different density.

A form for the time-scale of this relaxation is required. For this the approach of Gudiksen \& Nordlund (2005) is adopted. In simulating coronal heating they chose $\tau$ to depend on some power of the density

$\tau=\left(\frac{\rho}{\rho}_{\mathrm{ph}}\right)^{-1.7}$

so that at the relatively dense photosphere $\left(\rho=\rho_{\mathrm{ph}}\right)$ the timescale is about $0.1 \mathrm{~s}$ and is large enough that the effect becomes negligible in the sparse corona.

\subsection{The equation of state}

The specific energy density $\epsilon$ for a partially ionised hydrogen plasma containing ions, neutrals and electrons is given by

$\epsilon=\frac{P}{\rho(\gamma-1)}+\frac{n_{\mathrm{i}} \chi_{\mathrm{i}}}{\rho}$

where $P$ is the pressure, $\rho$ the density, $n_{\mathrm{i}}$ the number density of the ions, $\chi_{\mathrm{i}}$ the ionisation energy of hydrogen and $\gamma$ is the ratio of specific heats. Using the ideal gas law for the pressure

$P=n k_{\mathrm{B}} T$

where $T$ is the temperature and $n$ is the total number density $n=n_{\mathrm{i}}+n_{\mathrm{n}}+n_{\mathrm{e}}$, and using the definition of the neutral fraction

$\xi_{\mathrm{n}}=\frac{n_{\mathrm{n}}}{n_{\mathrm{n}}+n_{\mathrm{i}}}$

Eq. (42) can be written as

$\epsilon=\frac{k_{\mathrm{B}} T}{\mu_{\mathrm{m}}(\gamma-1)}+\left(1-\xi_{\mathrm{n}}\right) \frac{\chi_{\mathrm{i}}}{m_{\mathrm{i}}}$ 
were $\mu_{\mathrm{m}}$ is the reduced mass. The approximation $\rho \approx m_{\mathrm{i}} n_{\mathrm{i}}+$ $m_{\mathrm{n}} n_{\mathrm{n}}=m_{\mathrm{i}}\left(n_{\mathrm{i}}+n_{\mathrm{n}}\right)$ is used as the mass of the electron is small compared with the proton/neutron.

The MHD equations solve for the state variables $\epsilon$ and $\rho$ and so at any point in the main update the temperature $T$ and pressure $P$ must be derived from Eq. (45). This equation is actually a formula for $T$ in terms of $\epsilon$ and $\rho$, because the neutral fraction, $\xi_{\mathrm{n}}$ is a function of $T$ and $\rho$. Hence, in order to obtain the temperature, this equation must be solved for $T$ at every step, given the values of $\rho$ and $\epsilon$. The pressure at this time-step can then be found from

$P=n k_{\mathrm{B}} T=\frac{\rho k_{\mathrm{B}} T}{\mu_{\mathrm{m}}}$.

Equation (42) contains two terms, the gas pressure term and the ionisation term. In the photosphere and corona the gas pressure term is dominant, but in the partially ionised region of the lower chromosphere these terms are comparable, so both terms should be included in a complete simulation. However, the simulations presented here use only the gas term in Eq. (42) for a number of reasons. Firstly, this approach allows direct comparison with the previous emergence simulations in the literature (Magara \& Longcope 2001; Archontis et al. 2004; Shibata et al. 1989), which neglect both the effect of partial ionisation in the equation of state and the increased resistivity perpendicular to the field in the induction equation.

Secondly, although pressure gradients are important for the buoyant rise of flux tubes, the emergence of magnetic field through the atmosphere in these simulations is dominated by the enhanced dissipation of field through the partially ionised region, not buoyancy. As a result the exact nature of the pressure calculations is unimportant with regards to the results presented here, and the pressure term is not included in Eq. (42). However, the precise effect of the form of Eq. (42) on flux emergence is not known, and future work, which will include 3D simulations, will quantify this effect.

\section{Numerical method}

\subsection{Numerical code}

The modified MHD equations (Eqs. (27) to (30)) are solved numerically using the Lagrangian remap code Lare2d (Arber et al. 2001). The MHD equations, modified to include the possible presence of neutrals are presented below in Lagrangian form

$$
\begin{aligned}
\frac{\mathrm{D} \rho}{\mathrm{D} t} & =-\rho \nabla \cdot \boldsymbol{v} \\
\frac{\mathrm{D} \boldsymbol{v}}{\mathrm{D} t} & =-\frac{1}{\rho} \nabla P+\frac{1}{\rho} \boldsymbol{j} \wedge \boldsymbol{B}+\boldsymbol{g}+\nabla \cdot \boldsymbol{S} \\
\frac{\mathrm{D} \boldsymbol{B}}{\mathrm{D} t} & =(\boldsymbol{B} \cdot \nabla) \boldsymbol{v}-\boldsymbol{B}(\nabla \cdot \boldsymbol{v})-\nabla \wedge\left(\eta \boldsymbol{j}_{\|}\right)-\nabla \wedge\left(\eta_{\mathrm{c}} \boldsymbol{j}_{\perp}\right) \\
\frac{\mathrm{D} \boldsymbol{\epsilon}}{\mathrm{D} t} & =-\frac{P}{\rho} \nabla \cdot \boldsymbol{v}+\eta \dot{j}_{\|}^{2}+\eta_{\mathrm{c}} j_{\perp}{ }^{2}+\varsigma_{i j} S_{i j}-\frac{\epsilon-\epsilon_{0}(\rho)}{\tau}
\end{aligned}
$$

with the same definitions as in Sect. 2.

In $2.5 \mathrm{D}$ the density, specific energy density and magnetic field in the ignorable direction $(z)$ are defined at the centre of the grid. The velocities are defined at the vertices, while $\boldsymbol{B}_{\boldsymbol{x}}$ is defined on the right hand side edge and $\boldsymbol{B}_{\boldsymbol{y}}$ on the top edge of the cell. This staggered grid preserves $\nabla . \boldsymbol{B}$ in the simulation. The code uses linear and quadratic artificial viscosities to preserve shock structure moving obliquely across the grid (Arber et al. 2001).

\subsection{Implementing the induction equation for a partially ionised plasma}

The components of the current parallel and perpendicular to the magnetic field are calculated at each step by

$j_{\|}=\frac{(j . B) B}{|B|^{2}}$

$\boldsymbol{j}_{\perp}=\frac{\boldsymbol{B} \wedge(\boldsymbol{j} \wedge \boldsymbol{B})}{|\boldsymbol{B}|^{2}}$

respectively.

The value of $\eta$ is calculated from Eq. (11). $\eta_{\mathrm{c}}$ is evaluated at each step as a function of $x$ and $y$ from the local values of density, temperature and magnetic field using the equations for the ionisation degree (Eqs. (34) to (37)) and the relationship between the neutral fraction and $\eta_{\mathrm{c}}$ (Eq. (21)).

Khodachenko et al. (2004) showed that for certain magnetic field models, and using the VALC model for plasma variables that the ratio $\eta_{\mathrm{c}} / \eta$ can be orders of magnitude. Thus the value of $\eta_{\mathrm{c}}$ becomes the dominant effect on the condition for stability of the numerical solution to the induction equation

$\Delta t_{\text {diff }} \leq \frac{\Delta x^{2}}{\eta_{\mathrm{c}}} \leq \frac{\Delta x^{2}}{\operatorname{MAX}\left(\eta, \eta_{\mathrm{c}}\right)}$

where $\Delta x$ is the minimum cell size in either direction. Given typical values for the solar atmosphere, the condition for stability for the diffusion equation for a partially ionised plasma is more dominant than the CFL condition for advection associated with the ideal update.

$\Delta t_{\text {diff }}<\Delta t_{\text {adv }}$

where

$\Delta t_{\mathrm{adv}} \leq \frac{\Delta x}{c}$

Here $c$ is the maximum local velocity in the system.

To avoid subjecting the whole scheme to this diffusion time-step condition the resistive update for a partially ionised plasma should be done separately from the main update for ideal MHD. To do this the resistive update is sub-cycled inside each ideal step. For every ideal step, which is subject to the advective CFL condition, the resistive step is performed $n$ times, where

$n=I N T\left(\frac{\Delta t_{\mathrm{adv}}}{\Delta t_{\mathrm{diff}}}\right)+1$.

Now the Lagrangian step is not restricted by the smaller diffusion limit and this decreases the overall run time of the simulation, but also keeps the resistive update stable. This sub-cycling code was tested against the same code using the minimum of both time-step conditions for the entire scheme. There was no difference in the two sets of solutions and thus sub-cycling is both accurate and efficient. 


\subsection{Implementing the Newton cooling term}

The non-adiabatic terms in the energy equation due to mechanisms such as shock heating and thermal conduction are being modeled by using a Newton-cooling equation for the internal specific energy density.

$\frac{\mathrm{D} \epsilon}{\mathrm{D} t}=-\frac{\left(\epsilon-\epsilon_{0}(\rho)\right)}{\tau}$

where $\tau$ is the relaxation time-scale and as mentioned in Sect. 2.2 has been chosen to be negligible in the corona and most important at photospheric levels. To apply this relaxation to the internal specific energy density this equation must be solved numerically at each time-step in the code in addition to the MHD equations. A first order implicit finite difference method is used.

$\frac{\epsilon^{n+1}-\epsilon^{n}}{\mathrm{~d} t}=-\frac{\left(\epsilon^{n+1}-\epsilon_{0}(\rho)\right)}{\tau}$

where the superscript represents the discrete time sequence. The update for the specific energy density is then given by

$\epsilon^{n+1}=\frac{\epsilon^{n}+\frac{\mathrm{d} t}{\tau} \epsilon_{0}(\rho)}{1+\frac{\mathrm{d} t}{\tau}}$.

The reason for choosing the method shown is that it is unconditionally stable, Applying the standard Von-Neumman stability analysis the multiplication factor $g$ is always less than unity.

$g=\frac{1}{1+\frac{\mathrm{d} t}{\tau}}<1$.

\subsection{Initialisation}

The modified MHD Eqs. (27) to (30) are normalised by division of SI variables by photospheric values. The basic units are

$$
\begin{aligned}
y_{\mathrm{ph}} & =150 \mathrm{~km} \\
v_{\mathrm{ph}} & =6.5 \mathrm{~km} \mathrm{~s}^{-1} \\
\rho_{\mathrm{ph}} & =2.7 \times 10^{-4} \mathrm{~kg} / \mathrm{m}^{3}
\end{aligned}
$$

which gives the derived units

$t_{\mathrm{ph}}=23 \mathrm{~s}$

$T_{\mathrm{ph}}=6420 \mathrm{~K}$

$P_{\mathrm{ph}}=1.2 \times 10^{4} \mathrm{~Pa}$

$B_{\mathrm{ph}}=1200 \mathrm{G}$.

From here on unless stated all quoted values are internal code variables and should be multiplied by the above values to recover the SI variables.

The physical domain simulated extends vertically from -20 ( $3000 \mathrm{~km}$ below the surface) to $80(12000 \mathrm{~km}$ above). The horizontal extend is $50(7500 \mathrm{~km})$ about the centre of the domain.

The initial stratification is a simple 1D model of the temperature profile of the Sun, which includes the upper $3000 \mathrm{~km}$ of the convection zone, photosphere/chromosphere, transition zone, and corona. The temperature profile consists of a linear

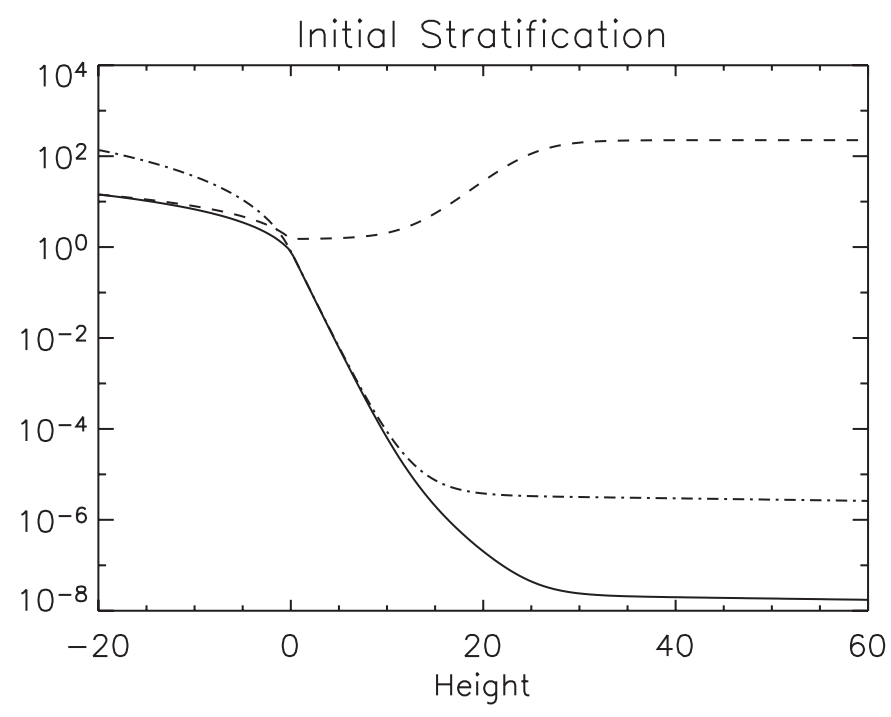

Fig. 2. Distribution of temperature (dashed line), density (solid line) and gas pressure (dot-dashed line) all values are normalised.

polytrope for the convection zone with a vertical gradient at the critical adiabatic value

$\frac{\mathrm{d} T}{\mathrm{~d} y}=\frac{\gamma-1}{\gamma} \frac{T}{P} \frac{\mathrm{d} P}{\mathrm{~d} y}$.

The temperature in the photosphere and chromosphere is assumed to be constant at 1 , as is the temperature in the corona at a temperature of 150 . These two regions are connected by a transition region of width $w_{\mathrm{tr}}=5$.

$$
\begin{aligned}
T(y) & =T_{\mathrm{ph}}-\frac{g}{m+1} y, y<0 \\
& =T_{\mathrm{ph}}+\frac{\left(T_{\mathrm{cor}}-T_{\mathrm{ph}}\right)}{2}\left[\tanh \left(\frac{y-y_{\mathrm{cor}}}{w_{\mathrm{tr}}}\right)+1\right], y>0
\end{aligned}
$$

$m=\frac{1}{\gamma-1}$ is the adiabatic index for a polytrope, $y_{\text {cor }}=25$ is the height of the corona, $T_{\mathrm{ph}}$ is the photospheric temperature and $T_{\text {cor }}=150$.

The density and gas pressure are specified by assuming hydrostatic equilibrium initially

$\frac{\mathrm{d} P}{\mathrm{~d} y}=-\rho g$

and the ideal gas law

$P=\rho T$

which is given in normalised form. Figure 2 shows the initial stratification in the normalised units. The numerical grid is uniform in the horizontal direction and stretched in the vertical. More cells are inserted in the centre of the domain where the flux tube emerges into the atmosphere, as this is the area of most interest As the sound speed increases towards the top of the domain, larger cells are used to keep the CFL condition (Eq. (55)) from unnecessarily slowing the running of the code. Using larger cells at the top of the domain is acceptable as there is no magnetic field so the solution is of little interest. A typical cell size near the flux tube is $\Delta x=\Delta y=0.2$. 
The evolution of a magnetic flux tube is initiated by inserting a uniformly twisted magnetic field profile in the convection zone, centred about $y=-12$.

$$
\begin{aligned}
& B_{z}=B_{0} \exp \left(-\frac{r^{2}}{a^{2}}\right) \\
& B_{\phi}=q r B_{r}
\end{aligned}
$$

where $r$ is the radial distance from the centre of the tube, $B_{z}$ is the magnetic field component in the axial direction $(z)$, and $B_{\phi}$ is the azimuthal component. The width of the tube $a$ is chosen to be 2, and the strength of the field at the centre of the tube, $B_{0}$, is 5. Following the approach of Archontis et al. (2004), the value of the twist, $q$, is chosen so that the tube is stable to drag in the convection zone which would otherwise fragment an untwisted tube (Moreno-Insertis \& Emonet 1996). In these simulations this corresponds to a value of $q=-1 / a$. This initialisation is a 2D cross-section of the 3D initialisation described in Fan (2001) and Archontis et al. (2004).

The plasma pressure inside the tube differs from the fieldfree pressure by $p_{1}(r)$, where

$$
\frac{\mathrm{d} p_{1}(r)}{\mathrm{d} r} \hat{\boldsymbol{e}_{r}}=\boldsymbol{j} \wedge \boldsymbol{B}
$$

so that the pressure gradient matches the Lorentz force. The change in density inside the flux tube relative to the field free atmosphere is specified by assuming the tube to be in thermal equilibrium with its surroundings.

$T_{0}(y)=\frac{p_{0}(y)}{\rho_{0}(y)}=\frac{p_{0}(y)+p_{1}(r)}{\rho_{0}(y)+\rho_{1}(r)}$.

The density inside the tube differs from the field-free atmosphere by

$\rho_{1}(r)=\frac{p_{1}(r)}{T_{0}(y)}$.

It is worth noting that this is only one method to initiate the rise of a flux tube in the convection zone. Other emergence simulations such as Magara (2001), use a force-free field inserted into a hydrostatic atmosphere. This equilibrium is then perturbed by vertical velocities for a short time to initiate the evolution of the flux tube. This initialisation is given the name mechanical equilibrium (MEQ). The method described in this paper is known as temperature balance (TBL). A third option as used by Moreno-Insertis \& Emnonet (1996) assumes equal entropy across the flux tube (EET).

Particular interest in this paper is taken in the nature of the force-free approximation. This approximation is used by many authors when dealing with coronal magnetic field, as the ratio of gas pressure to magnetic pressure, $\beta$, is low so that magnetic forces dominate and the equilibrium is given by $\boldsymbol{j} \wedge \boldsymbol{B}=0$. However below the surface of the Sun, where $\beta$ is greater than unity, magnetic fields cannot be assumed to be force-free. For this reason the MEQ method, which assumes a force-free subsurface magnetic field, is not used in these simulations. Instead a non-force-free field is used, which leaves two choices for the initialisation, TBL or EET. For simplicity, the TBL approach is used over the EET. It enables the tube to be made less dense

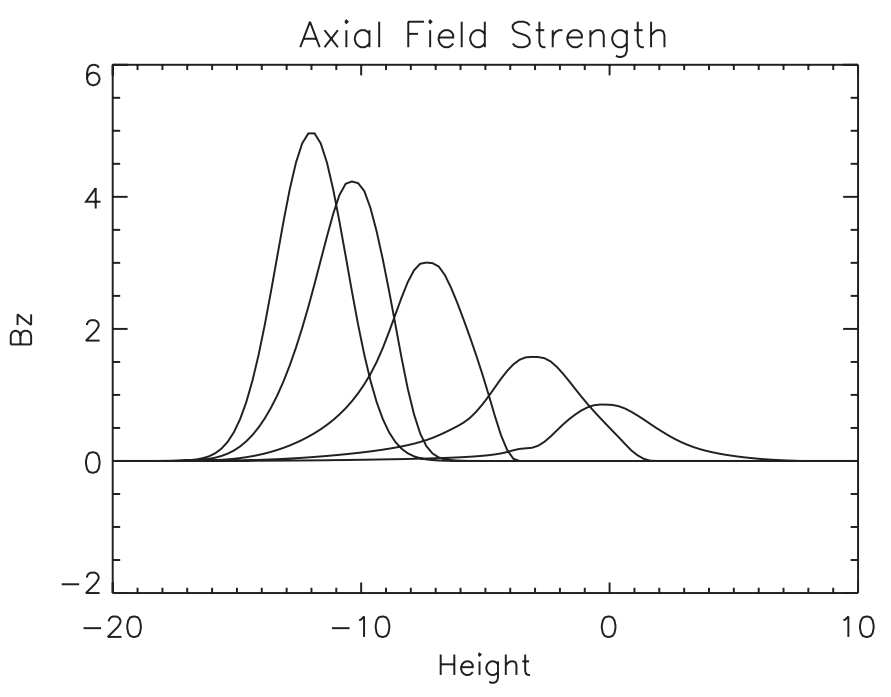

Fig. 3. Axial field strength as a function of height through the centre of the tube at $t=0,10,20,30,40$. All values are normalised to photospheric values.

than its surroundings and thus initiates buoyant rise in the convection zone.

The Coulomb resistivity $\eta$ is calculated from the model density and temperature and turns out to be less than roundoff in the code so all simulations are therefore run with $\eta=0$. The viscosity is set to 0.01 (internal units), while the linear and quadratic shock viscosities are both set to 0.5 . As the simulation domain has coronal conditions at the top and photospheric/convection zone conditions at the base, the left, right and top boundaries are open, allowing the free flow of plasma and magnetic flux out of the domain. The lower boundary is line-tied, so at each step the gradients in magnetic field, density and internal specific energy density are zero, while the velocities are set to zero. This most accurately represents the situation in the convection zone, where the plasma beneath is more dense.

\section{Results}

\subsection{Evolution of the magnetic field in the upper convection zone and photosphere}

The initially buoyant tube is driven vertically through the adiabatically stratified convection zone. This rise is similar to the 2.5D simulations of Emonet \& Moreno-Insertis (1998) and Magara (2001). The axial field strength decreases as the tube cross section increases during the rise, due to flux conservation inside the tube (Fig. 3).

$B_{z}(y)=B_{z}\left(y_{0}\right) \frac{\rho(y)}{\rho\left(y_{0}\right)}$.

As the flux tube meets the convectively stable photosphere, its vertical motion is halted and horizontal expansion spreads the flux tube out to form a contact layer with the plasma above (Fig. 4).

Now the majority of magnetic field is horizontal, i.e. $B_{x}$. This layer is holding up denser plasma than if there was no 

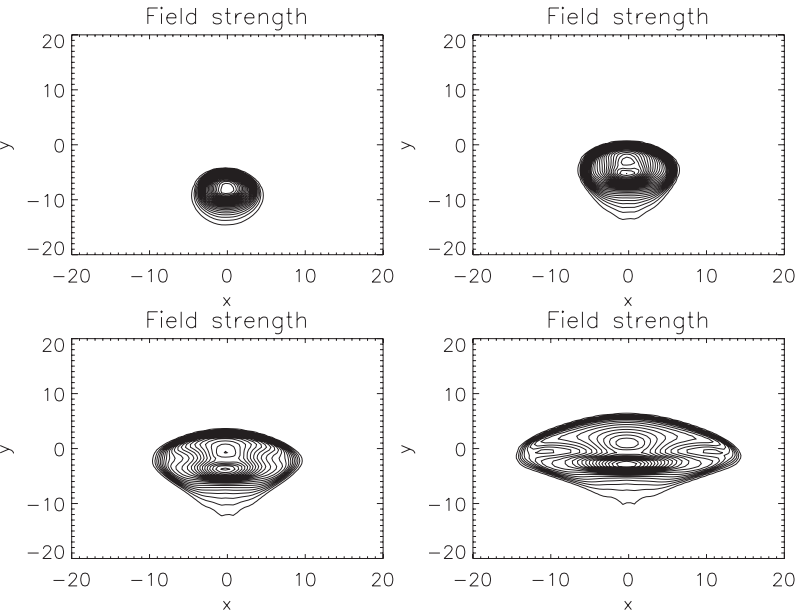

Fig. 4. Contours of magnetic field strength at times $t=20,30,35$, 40 showing the horizontal expansion of the tube as it meets the stable photosphere $(y=0)$.

magnetic field and is unstable to a Rayleigh-Taylor like instability known as the magnetic buoyancy instability (Archontis et al. 2004; Newcomb 1961; Thomas \& Nye 1975; Acheson 1979). Gilman (1970) derived criteria for the onset of this instability in an isothermal adiabatic atmosphere. The criteria for onset of the magnetic buoyancy instability can be regarded as a competition between the destabilising effect of the gradient in the magnetic field and the convectively stable temperature gradient.

For modes that do not twist field lines the criteria for onset is given by

$-\frac{g}{\gamma C_{\mathrm{s}}^{2}} \frac{\mathrm{d}}{\mathrm{d} y} \ln \left(\frac{B}{\rho}\right)>\frac{N^{2}}{C_{\mathrm{A}}{ }^{2}}$

while for modes that twist field lines the criteria is

$-\frac{g}{\gamma C_{\mathrm{s}}^{2}} \frac{\mathrm{d}}{\mathrm{d} y} \ln (B)>k^{2}\left(1+\frac{l^{2}}{n^{2}}\right)+\frac{N^{2}}{C_{\mathrm{A}}^{2}}$.

Here $B, \rho, \gamma$ have the usual meanings, $C_{\mathrm{A}}$ is the local Alfvén speed and $C_{\mathrm{s}}$ the local sound speed. $N$ is the Brunt-Wäisälä frequency given by

$N^{2}=\frac{g}{\gamma} \frac{\mathrm{d}}{\mathrm{d} y} \ln \left(\frac{p}{\rho^{\gamma}}\right)$

which is a measure of the convective stability of the atmosphere. $k, l$ and $n$ are the wave-numbers of the mode where $k$ is the wave number parallel to the magnetic field.

The criteria (Eq. (73)) can be rewritten as

$-H_{\mathrm{p}} \frac{\partial}{\partial y} \ln (B)>k^{2}\left(1+\frac{l^{2}}{n^{2}}\right)-\frac{\gamma}{2} \beta \delta$

where $H_{\mathrm{p}}$ is the local scale height and $\delta$ is called the superadiabatic excess, which is the difference between the logarithmic temperature gradient and its adiabatic value (Stix 2002) and is negative.

The stabilising term, which depends on the plasma beta $\beta$, prevents further rise of magnetic field above the contact layer.
After the upper part of the flux tube forms the horizontal magnetic layer at the base of the photosphere, more and more flux from the bottom of the tube is pushed up below it. Thus $\beta$ decreases and the stabilising effect of this term becomes less and less important until the instability sets in and magnetic field can expand into the atmosphere above. For the simulations here, the onset of the instability does occur when the local value of $\beta$ at the contact layer falls below the critical value, and the growth rates match that of the 2.5D simulations of Shibata et al. (1989) and Magara \& Longcope (2001).

\subsection{Effect of heating mechanisms in the lower atmosphere}

The effect of including heating phenomena in the solar atmospheric model is investigated by comparing the standard model with the model mentioned in Sect. 2.2 which forces the temperature in the lower atmosphere to relax to its initial value. The effects are investigated separately from the effects of partial ionisation which are undertaken in Sect. 4.3. Thus when investigating the effects of extra heating terms, the models are fully ionised and the only difference is in the energy equation.

The simple interchange derivation for the onset of the magnetic buoyancy instability assumes that as a parcel of gas moves upwards into its new surroundings, the motion is adiabatic and no heat transfer occurs. However by attempting to simulate the effects of various non-adiabatic terms in the energy equation the criteria for the instability to occur must be modified. Gilman (1970) applied the same argument as above but instead of assuming adiabatic motion, the displaced parcels of gas instantaneously acquire the temperature of their new surroundings (this corresponds to the thermal conductivity being infinite). The criteria for the onset of the magnetic buoyancy instability for modes that twist field lines is then

$-\frac{g}{\gamma C_{\mathrm{s}}{ }^{2}} \frac{\mathrm{d}}{\mathrm{d} y} \ln (B)>k^{2}\left(1+\frac{l^{2}}{n^{2}}\right)$.

It can be seen by comparison with Eq. (73) that the stabilising effect of the convectively stable gradient of the photosphere has been destroyed by this instantaneous heat transfer. In these simulations, although the heat transfer is not instantaneous, there is heat transfer on a finite time-scale, represented by the relaxation term for the energy (Eq. (40)).

It is expected that with the heat transfer present in these simulations the stabilising term in Eq. (73) will be reduced by some amount, just as for instantaneous transfer it was nullified completely. So the onset of the magnetic buoyancy instability should occur earlier in the simulation than in the adiabatic case. This is because less magnetic field is needed to decrease the effect of the competing stabilising term, which is now less important.

The magnetic field in the centre bends upwards and drags flux into the atmosphere. Thus to measure the growth of the instability the vertical velocity at the centre of the domain just above the magnetic layer at a height of $10(1500 \mathrm{~km})$ is plotted as a function of time. Figure 5 shows these plots for both the adiabatic case and the non-adiabatic case. As can be seen the non-adiabatic atmosphere allows onset of the instability much 


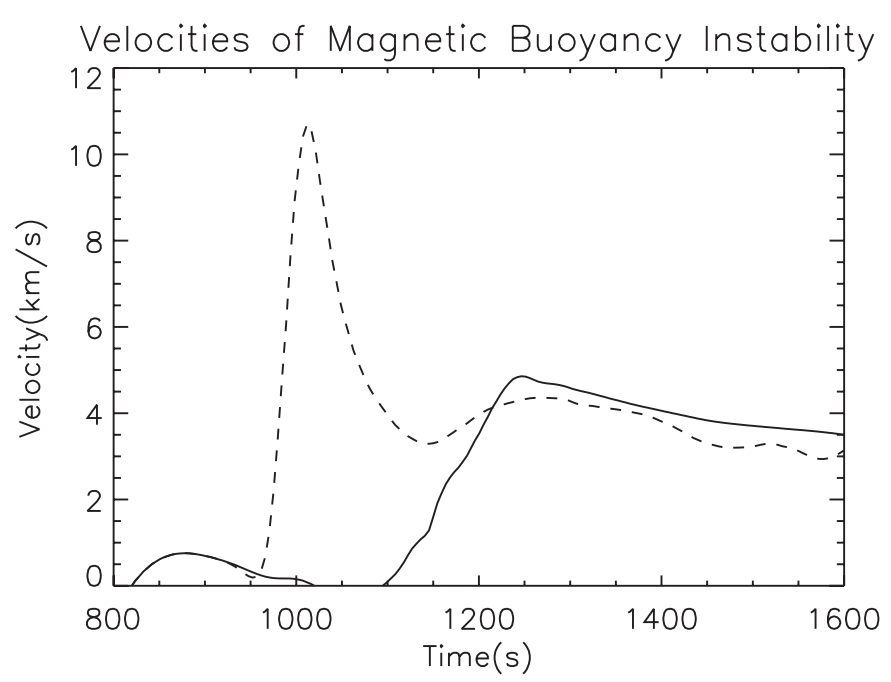

Fig. 5. Plasma velocity at a height of $1500 \mathrm{~km}$ above the surface as a function of time showing the onset of the magnetic buoyancy instability. The solid line is the adiabatic case and the dashed line is the non-adiabatic case.

earlier in the simulation. This is because less magnetic field has to build up to nullify the stabilising term in Eq. (73). The growth rates are also different, the one for the non-adiabatic case being larger. The instability quenches earlier for the nonadiabatic case. The magnetic field is emerging at a greater rate than the adiabatic situation and so the velocities at this height will decrease quicker to background values as the instability quenches.

As the magnetic buoyancy instability develops magnetic field expands into the chromosphere and corona above. As this happens cold dense plasma is brought up. Associated with this expansion is a cooling of the plasma, brought on by the $P \nabla . v$ term in the energy equation. Without any non-adiabatic terms such as heat conduction and radiative heating to counter this rapid cooling the resulting atmosphere would be unphysical. By modeling the effect of these non-adiabatic terms a more realistic atmosphere during emergence can be achieved.

Figure 6 shows the vertical temperature profile up the centre of the domain at $t=75$ in the simulation, representing the final stratification. Also shown is the initial profile from the model. In the adiabatic case the cold dense plasma brought up with the magnetic field has expanded and cooled to temperatures an order of magnitude lower than the initial photospheric temperature. By modeling the non-adiabatic terms using the relaxation method, the photospheric temperatures remain similar to the initial values as the heat transfer relaxes the temperature to its quasi-equilibrium.

As can be seen from Fig. 7, dense plasma is still being brought up into the atmosphere in both simulations. However when the effects of non-adiabatic heating are modeled this dense plasma is heated to its original temperature rather than being cooled as it expands. This is not only important in itself, but as will be shown in later sections, this is important for the calculation of other parameters in the simulations, such as the Cowling resistivity which depends on local values of $\rho$ and $\epsilon$.

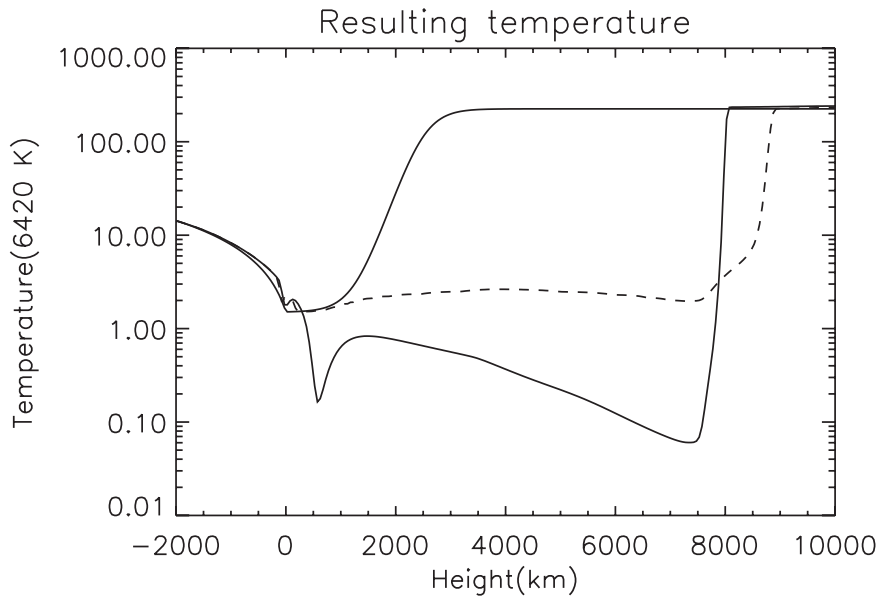

Fig. 6. Temperature as a function of height. The upper solid line shows the initial profile, the lower solid line is the resultant profile at $t=75$ for the adiabatic case, and the dashed line is for the simulation where non-adiabatic effects are included. The temperature is normalised to the photospheric value of $6420 \mathrm{~K}$.

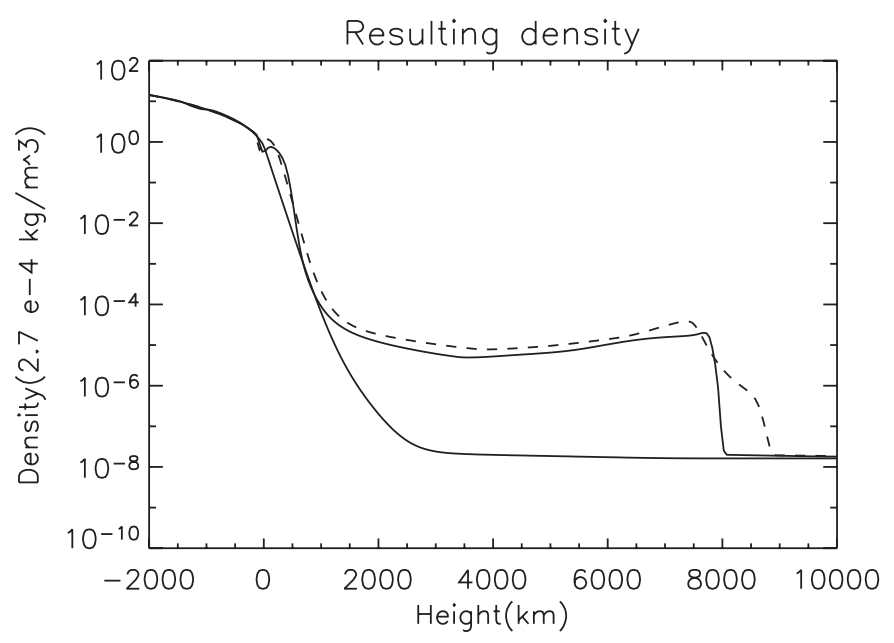

Fig. 7. Density as a function of height. The lower solid line shows the initial profile, the upper solid line is the resultant profile at $t=75$ for the adiabatic case, and the dashed line is for the simulation where non-adiabatic effects are included. The density is normalised to the photospheric value of $2.7 \times 10^{-4} \mathrm{~kg} / \mathrm{m}^{3}$.

\subsection{Effect of a partially ionised atmosphere}

The effect of including a partially ionised atmosphere when simulating flux emergence is investigated by comparing results for two simulations. The first is where the model is a fully ionised plasma (FIP). This means $\eta_{\mathrm{c}}$ is zero and so the standard MHD equations apply. The second is where the model contains the partially ionised plasma in the chromosphere (PIP). Here, the ionisation level and $\eta_{\mathrm{c}}$ are evaluated as functions of the local density, temperature and magnetic field strength.

For a partially ionised plasma $\eta_{\mathrm{c}}$ dissipates cross-field currents. As the simulation progresses the local density and temperature change and thus so does the local value of $\eta_{\mathrm{c}}$. In Sect. 4.2 it was shown that by modeling the effects of various heat transfer mechanisms, the atmosphere reacted to the emerging plasma without the vast cooling seen when the 


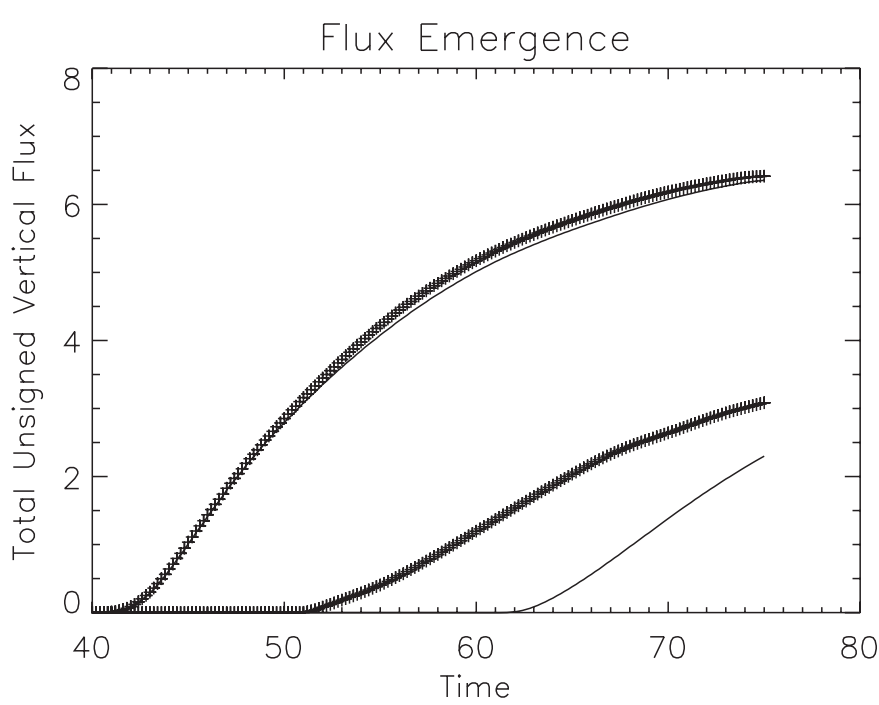

Fig. 8. Total unsigned flux at constant heights as a function of time in the simulation. The two plots on the left are at heights $500 \mathrm{~km}$, and the two plots on the right are at $2500 \mathrm{~km}$ above the surface. The solid lines are for the FIP model and the crosses are for the PIP model.

effects were ignored. Thus the change in $\eta_{\mathrm{c}}$ during the simulation represents a realistic variation during the formation of an active region. For all following simulations, the Newtoncooling approach described in Sect. 3.3 is applied.

\subsubsection{Emergence of magnetic flux}

The profile of $\eta_{\mathrm{c}}$ (Fig. 1) represents a diffusive region of approximately 10 scale heights. As the magnetic buoyancy drives magnetic flux and plasma through the model chromosphere and into the corona, the presence of a diffusive layer in the chromosphere should affect the rate of emergence of this flux. By comparing the amount of flux emerging through various heights of the atmosphere for the two models, the importance of this layer can be evaluated. This is done using the amount of unsigned vertical flux

$\int\left|B_{y}\right| \mathrm{d} x$

where the integral is over the horizontal extent of the domain. Figure 8 shows the total flux emerging at two different heights for the two models, as a function of time. The lower height is $500 \mathrm{~km}$, in the photosphere below the diffusive layer, and the upper height is $2500 \mathrm{~km}$, above the diffusive layer. At the lower height the flux emerging for the FIP model (crosses) and the PIP model (solid) are almost identical, as at this height $\eta_{\mathrm{c}}=0$. At $2500 \mathrm{~km}$ the flux emerging is greatly increased by the presence of the diffusive layer in the PIP model (crosses) compared to the FIP model (solid line). As the magnetic field passes through this layer it is diffused on a time-scale

$t_{\mathrm{d}}=\frac{L^{2} \mu_{0}}{\eta_{\mathrm{c}}}$

where $L$ is the vertical extent of the region. The profile of $\eta_{\mathrm{c}}$ is changing during the simulation as the plasma variables change. Using the maximum values of $\eta_{\mathrm{c}}$ in these simulations, gives
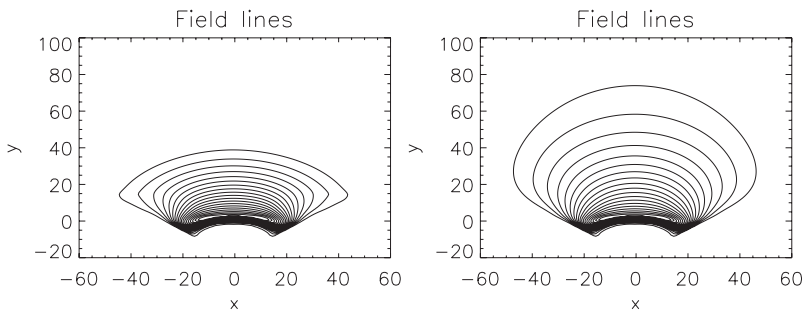

Fig. 9. Field-lines in $x$ and $y$ given by contours in $A_{z}$ where $\boldsymbol{B}=\nabla \wedge \boldsymbol{A}$. The left panel is the FIP model, the right is the PIP model. Both plots use the same contour levels.

a diffusive time-scale of $t_{\mathrm{d}} \approx 5 \mathrm{~s}$ which compares to the local transit time of flux across this region of $t_{\mathrm{t}} \approx 500 \mathrm{~s}$. Hence the magnetic field takes many diffusive time-steps to transit the partially ionised layer. Hence, locally, the magnetic Reynolds number (using $\eta_{\mathrm{c}}$ rather than the standard definition of resistivity) is well below unity, even though the majority of the domain is high Reynolds number.

The resultant magnetic field structure is therefore different for the two models. Figure 9 shows 2D field-lines in $x$ and $y$ for the two different models. The field-lines are given by the contours in $A_{z}$ where $\boldsymbol{B}=\nabla \wedge \boldsymbol{A}$. The resultant field is more diffuse in the PIP model, and the field-lines extend higher into the atmosphere, which is as expected when an extra diffusive layer is added. The inclusion of the presence of neutrals in the simulation of emerging flux yields more rapid emergence and a greater amount of flux in the corona.

\subsubsection{Cross-field and field-aligned currents}

Observations of magnetic fields in the solar atmosphere suggest that the solar corona is predominantly force-free (Georgoulis \& Labonte 2004). The low $\beta$ plasma of the solar corona is magnetically dominated, and the pressure and gravity terms in the momentum equation (Eq. (28)) are small in comparison to the Lorentz force. The equilibrium equation is then given by

$\boldsymbol{j} \wedge \boldsymbol{B}=0$.

This is equivalent to saying that the current is aligned with the magnetic field, $\boldsymbol{j} \| \boldsymbol{B}$, or that there are no cross-field currents, $j_{\perp}=0$.

Beneath the surface the plasma $\beta$ is much greater than unity, and therefore pressure forces dominate magnetic forces. The field in this region cannot be assumed to be force-free. If indeed, active regions are the product of emerging sub-surface field as evidence suggests (Zwann 1977), then the force-free coronal field must be formed from non force-free fields. This raises an important question. How is the magnetic field of subsurface flux tubes converted into force-free magnetic coronal field?

As stated in Sect. 2.1, for a partially ionised plasma, the current is not dissipated isotropically. The Coulomb resistivity $\eta$ acts parallel to the field, and $\eta_{\mathrm{c}}$ acts perpendicular to it (Eq. (25)). However, it has been shown that for the solar chromosphere $\eta_{\mathrm{c}} / \eta$ can be orders of magnitude. In fact $\eta$ is less than numerical round-off throughout the whole domain for these simulations. So the approximation $\eta=0$ is reasonable. This 


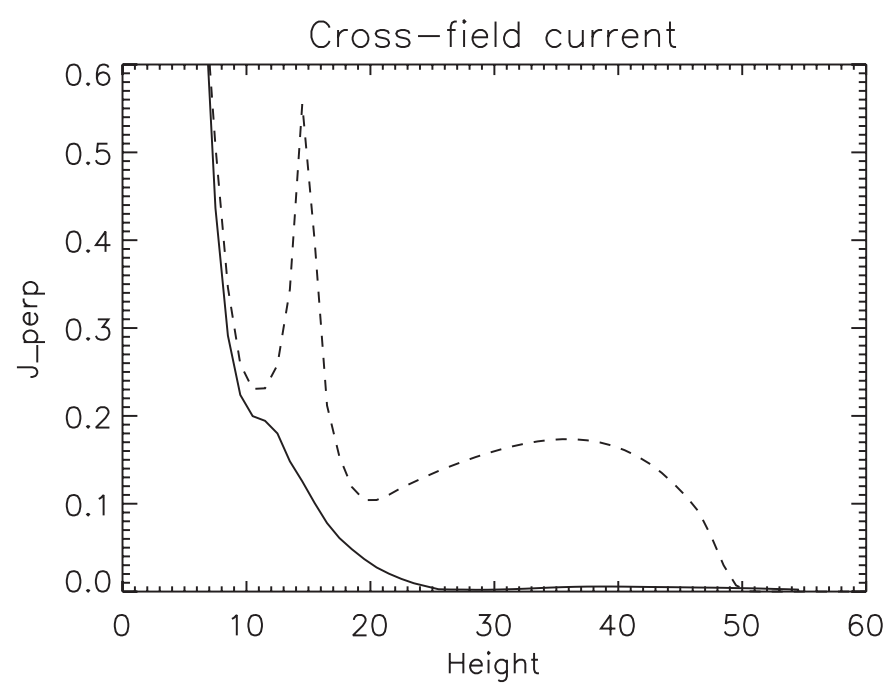

Fig. 10. Total perpendicular current across the domain as a function of height for the FIP model (dashed line) and the PIP model (solid line). All values are given in normalised units.

means that as the magnetic field emerges through the partially ionised region of the model atmosphere, the only component of the current to be dissipated directly is the cross-field current.

This gives a mechanism for the formation of force-free current during flux emergence. As magnetic field is driven though the partially ionised layer by the magnetic buoyancy instability, the energy of cross-field currents are dissipated by the ionneutral interactions. While the field-aligned currents are not directly affected. Hence if the value of $\eta_{\mathrm{c}}$ is large enough the field that emerges through the partially ionised region will have no cross-field currents and will therefore be force-free.

By comparing the amount of cross-field current emerging into the corona for the two models, the efficiency of this mechanism in forming force-free currents can be investigated. A measurement of the amount of cross-field current is obtained by performing the integral

$J_{\perp}(y)=\int\left|\boldsymbol{j}_{\perp}(x, y)\right| \mathrm{d} x$,

over the horizontal extent of the domain.

Figure 10 shows this as a function of height at $t=75$, for both the fully ionised model (dashed line) and the PIP model. The fully ionised plasma does not destroy cross-field currents and they are allowed to emerge into the corona with the magnetic field, giving a non force-free configuration. However, when the field emerges through a partially ionised plasma, nearly all of the cross-field current is destroyed, which corresponds to a force-free magnetic field. Figure 11 shows the integral

$J_{\|}(y)=\int\left|\dot{j}_{\|}(x, y)\right| \mathrm{d} x$

as a function of height at $t=75$ for both models.

Although $\eta=0$, there is a difference in $J_{\|}(y)$ for the two models. The equation for $\boldsymbol{j}_{\|}$and $\boldsymbol{j}_{\perp}$ are coupled to each other, and as the two models have different values of $\eta_{\mathrm{c}}$, it is expected that the amount of $\boldsymbol{j}_{\|}$emerging will differ slightly. Evidently,

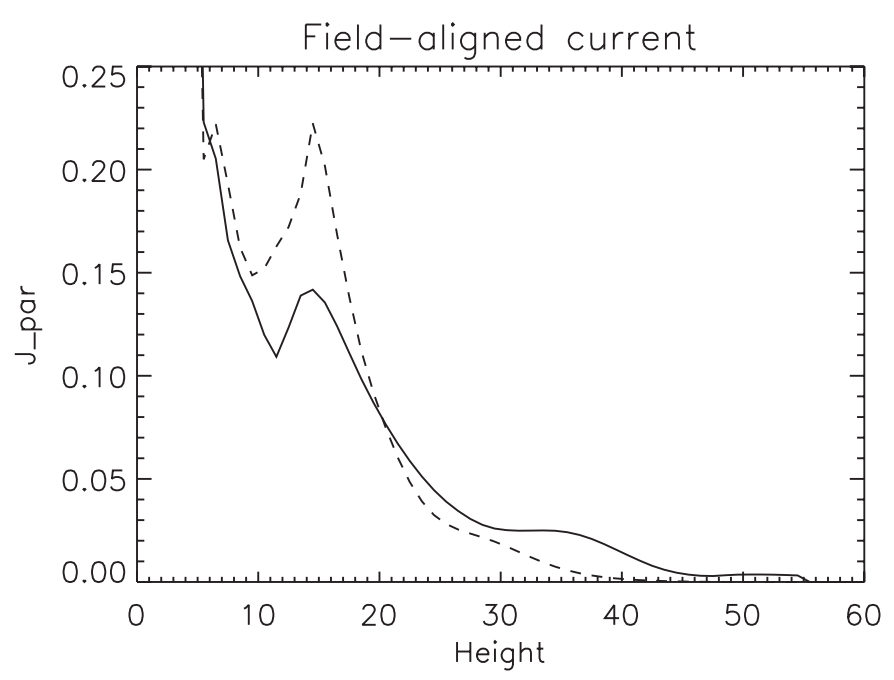

Fig. 11. Total parallel current across the domain as a function of height for the FIP model (dashed line) and the PIP model (solid line). All values are given in normalised units.

the presence of a partially ionised region in the solar atmosphere has destroyed the cross-field current but allowed the same amount of field-aligned current to emerge as in the case for a fully ionised plasma. Hence the field has been converted from a general configuration to one approaching a force-free state.

Calculations of the force-free nature of atmospheric magnetic field have been made based on observations using MDI (Georgoulis \& Labonte 2004; Metcalf et al. 1995). Vector magneto-grams have been extrapolated to reconstruct coronal field. Estimates of the height at which the field becomes forcefree are typically $400-1000 \mathrm{~km}$ above the photosphere. A typical height for these simulations based in Fig. 10 is much larger than this, around $2000 \mathrm{~km}$. This discrepancy may lie in the $2 \mathrm{D}$ nature of the simulations, and further 3D work will be performed to better diagnose the height at which field becomes force-free. However, these results show that in 2D at least, the emerging field is not force-free unless the partially ionised regions of the atmosphere are included in the models.

Throughout these simulations the Hall term has been neglected from Ohm's law. Figure 12 shows the magnitudes of the Hall term $\left(\left|\frac{j \wedge \boldsymbol{B}}{e n_{\mathrm{e}}}\right|\right)$, the advection term $(|\boldsymbol{v} \wedge \boldsymbol{B}|)$ and the diffusive term for ion-neutral collisions $\left(\left|\eta_{\mathrm{c}} \boldsymbol{j}_{\perp}\right|\right)$, as functions of height at the centre of the horizontal domain for a simulation run at $t=75$. These plots represent typical values for the entire domain. The Hall term is always orders of magnitude lower than the advection term. Figure 12 also shows that the magnetic Reynolds number for the partially ionised plasma can reach values less than unity.

\section{Conclusions}

2.5D simulations of the emergence of magnetic flux into the solar atmosphere have been performed. The standard model used was that of a weakly twisted flux tube inserted into an adiabatically stratified convection zone. The atmosphere of the Sun was modeled by two isothermal layers, representing the 


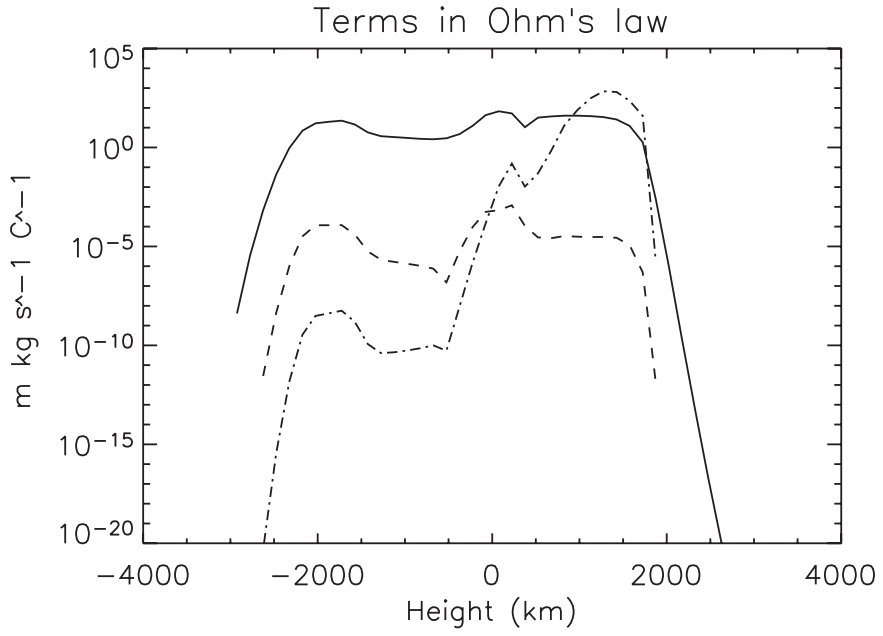

Fig. 12. Vertical profile of terms in Ohm's law in the centre of the horizontal domain at $t=75$. The solid line is the advection term, the dashed line is the Hall term, and the dash-dot line is the diffusive term due to ion-neutral collisions.

photosphere/chromosphere and corona, separated by a transition region. The flux tube evolution was initiated by dropping the density inside to start a buoyant rise to the surface. The resultant evolution agrees with previous work of flux tubes in the convection zone (Magara \& Longcope 2001; Fan 2001).

Having briefly investigated the emergence mechanism, we then added to the standard model the effects of heating/cooling mechanisms in the solar atmosphere. Rather than simulate these effects (such as radiative heating, thermal conduction, and shock heating) directly in the model equations, we used a simple approach to model their effects. Direct simulation of these effects is difficult in large scale models, and as stated, not all heating mechanisms are known well enough to simulate directly. All these mechanisms, both known and unknown, give the temperature we observe through emission lines (Vernazza et al. 1981). Thus, a simple ad-hoc approach is to relax the temperature to these observed values, or in the case of these simulations, the model temperature profile. This was done by a Newton-cooling like term in the equations.

By modeling these heating/cooling mechanisms, we showed that the criteria for onset of the magnetic buoyancy instability was modified. The onset of the instability, which drives flux into the corona, occurred earlier. Also the resultant rate of expansion of magnetic field into the corona was larger for this new model. More importantly the reaction of the plasma to the expansion was much more realistic. In the adiabatic case, there was no competing terms to the cooling of plasma from $P \nabla . v$ expansion. This lead to vastly lower temperatures of the photospheric plasma, which is unrealistic. By including heating/cooling effects, the expansion of the plasma as the magnetic buoyancy instability develops did not lead to unrealistic cooling when dense plasma was brought up through the photosphere. The resultant temperature was more realistic for this new model.

As stated in Sect. 2.3, the equation of state used here does not include the ionisation term in the equation of state (Eq. (42)). A simpler equation of state was used to allow direct comparison with the literature, which are ideal MHD simulations, and use no resistivities in their simulations. The main results of this paper, concerning flux emergence and current dissipation, should be unaffected by the choice in equation of state, as they are not directly affected by pressure gradients.

The next set of simulations investigated the effect of the partially ionised regions of the solar atmosphere. The relatively low temperatures in the chromosphere mean that the fully ionised approximation used in standard MHD is not valid everywhere in the simulation domain. The model was modified to include the effects of a partially ionised region. Assuming a pure hydrogen plasma, we derived ionisation and neutral fractions. The MHD equations were then modified using a threefluid approach to Ohm's law.

For a partially ionised plasma, the Coulomb resistivity $\eta$ acts parallel to the magnetic field, and the Cowling resistivity $\eta_{\mathrm{c}}$ acts perpendicular to the magnetic field. Using the model values for density and temperature, $\eta$ and $\eta_{\mathrm{c}}$ were evaluated locally. $\eta$ was effectively zero throughout the whole simulation domain, whereas $\eta_{\mathrm{c}}$ was largest at heights of $1500 \mathrm{~km}$ above the surface.

We investigated the effect on the emergence of magnetic flux for the fully ionised (FIP) and partially ionised (PIP) plasma models. The profile of $\eta_{\mathrm{c}}$ represented a large diffusive region, and as the magnetic field expanded throughout this region, the field became more diffuse than the FIP case, where $\eta_{\mathrm{c}}=0$. The resultant field structure was different, with fieldlines extending further into the model corona for the PIP model, as the field was diffused more then the FIP magnetic field.

As already mentioned, coronal field is predominantly forcefree, due to the low value of $\beta$, the ratio of gas pressure to magnetic pressure. However, field originating in the high $\beta$ convection zone is not necessarily force-free. The main thrust of this work was to provide a mechanism for the conversion of non-force free sub-surface field into force-free coronal field. To do this we examined the amount of cross-field current emerging as the field expanded into the corona. For the FIP model, substantial cross-field currents emerged into the solar corona. However, in the PIP model less cross-field currents emerged.

The presence of neutrals in the PIP model lead to a large resistivity $\eta_{\mathrm{c}}$ perpendicular to the field, and so reduced the amount of cross-field currents emerging. Conversely $\eta=0$ meant that similar amounts of field aligned current emerged in both the FIP and PIP models. We suggest that the presence of neutrals in a partially ionised model of the atmosphere is very important when investigating the nature of the emerging magnetic field. The ion-neutral collisions in the plasma cause an increased resistivity perpendicular to the field. This is a viable mechanism for the conversion of a general field configuration to one approaching force-free as it preferentially dissipates cross-field currents.

The work presented here is a first attempt at simulating flux emergence through a partially ionised solar atmosphere. The initial stratification is an over simplified model of the Sun, with no pre-existing convection beneath the surface. In addition these results are from 2.5D simulations. To fully understand flux emergence it is necessary to work in 3D. The importance of shear forces and plasma draining in rising flux tubes 
has already been shown to be important for flux emergence (Manchester 2001; Manchester et al. 2004), as has the interaction with pre-existing coronal field (Archontis et al. 2004). Future work will have to include all these along with the methods applied in this work. Also the effect of joule heating, as given by $\eta_{\mathrm{c}} j_{\|}^{2}$, needs to be investigated relative to the other dynamics and thermal effects involved.

Acknowledgements. This work was funded in part by the Particle Physics and Astronomy Research Council (PPA/S/S/2003/03728).

\section{References}

Abbet, W. P., \& Fisher, G. H. 2003, ApJ, 582, 475

Acheson, D. J. 1979, SoPh, 62, 23

Ambartsumyan, V. A. 1958, Theoretical Astrophysics (Pergamon Press)

Arber, T. D., Longbottom, A. W., Gerrard, C. L., \& Milne, A. M. 2001, JCP, 171, 151

Archontis, V., Moreno-Insertis, F., Galsgaard, K., Hood, A., \& O'Shea, E. 2004, A\&A, 426, 1047

Berton, R. 2000, A\&A, 356, 301

Braginskii, S. I. 1965, Transport processes in a Plasma, in Reviews of plasma physics, Vol. 1 (New York: Consultants Bureau)

Brown, J. 1973, SoPh, 29, 421B

Caligari, P., Moreno-Insertis, F., \& Schüssler, M. 1995, ApJ, 441, 886

Caligari, P., Schüssler, M., \& Moreno-Insertis, F. 1998, ApJ, 502, 481

Cowling, MagnetoHydroDynamics, Monographs on Astronomical Subjects (Adam Hilger), 1957

D'Silva, S., \& Choudhuri, A. R. 1993, 272, 621

De Pontieu, B. 1999, A\&A, 347, 696

Emonet, T., \& Moreno-Insertis, F. 1998, ApJ, 492, 804

Fan, Y., Fisher, G. H., \& McClymont, A. N. 1994, ApJ, 436, 907

Fan, Y., Zweibel, E. G., Linton, M. G., \& Fisher, G. H. 1998, ApJ, 505, L59

Fan, Y. 2001, ApJ, 554, L111

Galsgaard, K., Moreno-Insertis, F., Archontis, V., \& Hood, A. 2005, ApJ, 618, L153

Georgoulis, M. K., \& LaBonte, B. J. 2004, 615, 1029

Gilman, P. A. 1970, ApJ, 162, 1019
Gilman, P. A., Morrow, C. A., \& DeLuca, E. E. 1989, ApJ, 338, 528

Goodman, M. L. 2000, ApJ, 533, 501

Gudiksen, B. V., \& Nordlund, A. 2005, ApJ, 618, 1020

Hughes, D. W., \& Proctor, M. R. E. 1988, Annu. Rev. Fluid. Mech., 20, 187

Khodachenko, M. L., \& Zaitsev, V. V. 2002, Ap\&SS, 279, 389

Khodachenko, M. L., Arber, T. D., Rucker, H. O., \& Hanslmeier, A. 2004, 422, 1073

Magara, T. 2001, ApJ, 549, 608

Magara, T., \& Longcope, D. W. 2001, ApJ, 559, 55

Magara, T., \& Longcope, D. W. 2003, ApJ, 586, 630

Manchester, W., IV 2001, ApJ, 547, 503

Manchester, W., IV, Gombosi, T., DeZeeuw, D., \& Fan, Y. 2004, ApJ, 610,588

Martinez-Pillet, V., Lites, B. W., \& Skumanich, A. 1997, ApJ, 474, 810

Matsumoto, R., \& Shibata, K. 1992, PASJ, 44, 167

Matsumoto, R., Tajima, T., Chou, W., Okubo, A., \& Shibata, K. 1993, ApJ, 414, 357

Metcalf, T. R., Jiao, L., McClymont, A. N., \& Canfield, R. C. 1995, 439,474

Moreno-Insertis, F. 1986, A\&A, 166, 291

Moreno-Insertis, F., \& Emonet, T. 1996, ApJ, 472, L53

Narain, U., \& Ulmschneider, P. 1990, SSRv, 54, 377

Newcomb, W. A. 1961, Phys. Fluids, 4, 391

Nozawa, S., Shibata, K., Matsumoto, R., et al. 1992, ApJS, 78, 267

Parker, E. N. 1954, ApJ, 121, 491

Parker, E. N. 1979, ApJ, 230, 905

Pottasch, S. R., \& Thomas, R. N. 1959, ApJ, 130, 941

Shibata, K., Tajima, T., \& Steinolfson, R. S. 1989, ApJ, 345, 584

Spiegel, E. A., \& Weiss, N. O. 1980, Nature, 287, 616

Spitzer, L. 1962, Physics of fully ionised gases (New York: Interscience)

Stix, M. 2002, The Sun: An Introduction (Springer)

Thomas and Athay 1961, Physics of the Solar Chromosphere, 3rd ed. (Oxford University Press)

Tomas, J. H., \& Nye, A. H. 1975, Phys. Fluids, 18, 490

Vernazza, J. E., Avrett, E. H., \& Loeser, R. 1981, ApJS, 45, 635

Wedemeyer 2004, A\&A, 414, 121

Zwann, C. 1977, SoPh, 60, 213 